\title{
Fractional Fourier Transform-Exceeding the Classical Concepts of Signal's Manipulation ${ }^{1}$
}

\author{
Z. Zalevsky ${ }^{a}$, H. M. Ozaktas ${ }^{b}$, and A. M. Kutay ${ }^{b}$ \\ ${ }^{a}$ School of Engineering, Bar-Ilan University, 52900, Ramat-Gan, Israel \\ ${ }^{b}$ Departament of Electrical Engineering, Bilkent University, TR-06800 Bilkent, Ankara, Turkey \\ Received December 28, 2006
}

\begin{abstract}
The fractional Fourier transform is a signal processing tool which is strongly associated with optical data manipulation. It has fast computational algorithms and it suggests solutions to interesting signal processing tasks. In this paper we review its properties as well as present a new set of its applications for blind source separation of images and for RF photonics (a field in which photonic devices are used to process RF signals).
\end{abstract}

PACS numbers: $02.30 . \mathrm{Nw}, 42.30 . \mathrm{Kq}$

DOI: $10.1134 / \mathrm{S} 0030400 \mathrm{X} 07120065$

\section{BASIC BACKGROUND}

The fractional Fourier transform (FRT) operation has been shown to be useful for various spatial filtering and signal processing applications [1-8]. The FRT is a private case of the $A B C D$ matrix. When the $A B C D$ matrix accepts the form

$$
A=D=\cos \phi, \quad B=-C=-\sin \phi .
$$

In this transform, the amount of shift variance may be controlled by choosing the proper fractional order $p$ for the transformation while $\phi$ equals to $\phi=\pi p / 2$. When the fractional order is one, the FRT becomes the conventional Fourier transform which is totally shift invariant. For fractional order of zero the FRT gives the input function, i.e., totally shift variant. For any other fractional order in between, the transform has partial amount of shift variance.

\subsection{Definition}

There are two common interpretations for the FRT. Both definitions were proven to be identical as shown in [7].

1.1.1. Definition based on propagation in graded index media. The first FRT definition [9-11] is based on the field propagating along a quadratic graded index (GRIN) medium having a length proportional to $p$ ( $p$ being the FRT order). The eigenmodes of quadratic GRIN media are the Hermite-Gaussian (HG) functions, which form an orthogonal and complete basis set. The $m$ th member of this set is expressed as

$$
\Psi_{m}(x)=H_{m}(\sqrt{2} x / \omega) \exp \left(-x^{2} / \omega^{2}\right),
$$

\footnotetext{
${ }^{1}$ The text was submitted by the authors in English.
}

where $H_{m}$ is a Hermite polynomial of order $m$ and $\omega$ is a constant associated with the GRIN medium parameters. An extension to two lateral coordinates $x$ and $y$ is straightforward, with $\Psi_{m}(x) \Psi_{n}(y)$ as elementary functions.

The propagation constant for each HG mode is given by

$$
\beta_{m}=k \sqrt{1-\frac{2}{k} \sqrt{\frac{n_{2}}{n_{1}}}\left(m+\frac{1}{2}\right)} \approx k-\sqrt{\frac{n_{2}}{n_{1}}}\left(m+\frac{1}{2}\right),
$$

where $k=2 \pi n_{1} / \lambda$, is the wavenumber and $n_{1}, n_{2}$ are related to the refraction index profile within the GRIN fiber as follows:

$$
n(r)^{2}=n_{1}^{2}\left[1-\left(n_{2} / n_{1}\right) r^{2}\right],
$$

$r$ is the radial coordinate. The HG set can be used in order to decompose any arbitrary distribution $u(x)$ as follows:

$$
u(x)=\sum_{m} A_{m} \Psi_{m}(x),
$$

where the coefficient $A_{m}$ of each mode $\Psi_{m}(x)$ is given by

$$
A_{m}=\int_{-\infty}^{\infty} u(x) \frac{\Psi_{m}(x)}{h_{m}} d x
$$

with

$$
h_{m}=2^{m} m ! \sqrt{\pi}(\omega / \sqrt{2}) .
$$


Using the above decomposition, the FRT of order $p$ is defined as

$$
\mathfrak{\Im}^{p}[u](x)=\sum_{m} A_{m} \Psi_{m}(x) \exp \left[i \beta_{m} p L\right],
$$

where

$$
L=(\pi / 2) \sqrt{n_{1} / n_{2}}
$$

is the GRIN length that results in the conventional Fourier transform. It was shown in [10] that this definition agrees well with the classical Fourier transform definition for the case of $p=1$.

\subsubsection{Definition based on Wigner distribution} function. A complete signal representation, displaying time and frequency information simultaneously, can be achieved by the time-frequency Wigner distribution function $(W D F)$. In [12] the FRT operation is defined by following the signal $u(x)$, while its $W D F$ is rotated by an angle $\phi$, which is related to the fractional order as follows: $\phi=p \pi / 2$. Note that the $W D F$ of a 1D function is a $2 \mathrm{D}$ function and the rotation interpretation is easily displayed. In [12], the same rotation strategy was generalized to $2 \mathrm{D}$ signals, i.e., images, whose WDFs are $4 \mathrm{D}$ distributions. The $W D F$ of a function can be rotated with bulk optics. It was suggested [12] to use the optical system of Fig. 1 for the implementation of the FRT operator following this definition.

This optical setup represents in the WDF space, the following three shearing operations: $x, v, x$ shearing or $v, x, v$ shearing, where $v$ and $x$ are the spectral and spatial coordinates, respectively. The $x$ shearing is performed by free-space propagation, followed by a lens which performs $v$ shearing, and another $x$ shearing which is performed by free-space propagation. In his paper [12], Lohmann characterized this optical system using two parameters, $Q$ and $R$, as follows:

$$
f=f_{1} / Q, \quad z=f_{1} R,
$$

where $f_{1}$ is an arbitrary length, $f$ is the focal length of the lens, and $z$ is the distance between the lens and the input (or output) plane. As known from [12] for an FRT of order $p, Q$, and $R$ should be chosen as follows:

$$
R=\tan (\phi / 2), \quad Q=\sin \phi
$$

for the type I configuration or

$$
R=\sin \phi, \quad Q=\tan (\phi / 2)
$$

for the type II configuration. Note that $\phi=p \pi / 2$. By analyzing the optical configuration of Fig. 1, Lohmann [12] obtained the following:

$$
\begin{gathered}
u_{p}(x)=\mathfrak{\Im}^{p}\left[u\left(x_{0}\right)\right] \\
=C_{1} \int_{-\infty}^{\infty} u\left(x_{0}\right) \exp \left(i \pi \frac{x_{0}^{2}+x^{2}}{\lambda f_{1} \tan \phi}\right) \exp \left(-2 \pi i \frac{x x_{0}}{\lambda f_{1} \sin \phi}\right) d x_{0}
\end{gathered}
$$

(a)

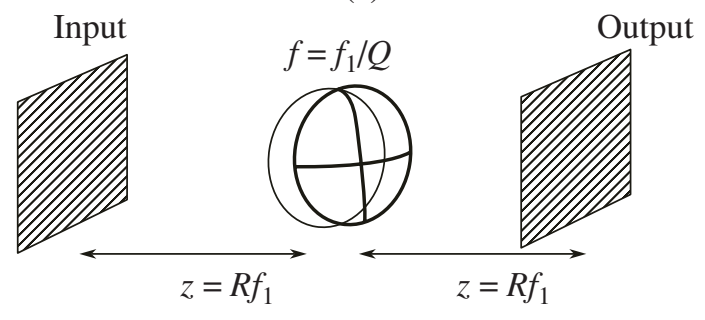

(b)

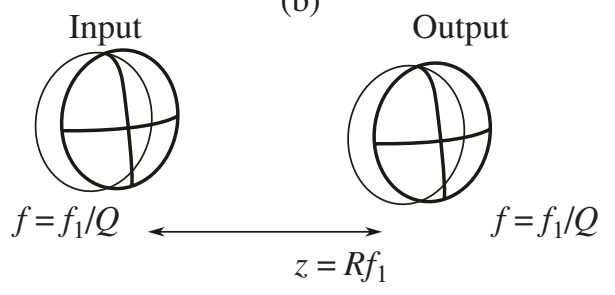

Fig. 1. The two possible optical setups for obtaining the FRT. (a) Type I configuration; (b) type II configuration.

with

$$
C_{1}=\exp [-i(\pi \operatorname{sgn}(\sin \phi) / 4-\pi / 2)] /\left|\lambda f_{1} \sin \phi\right|^{1 / 2} \text {. }
$$

This last equation defines the FRT for 1D functions where $\lambda$ is the wavelength. Generalization for $2 \mathrm{D}$ functions is straightforward. Note that $\lambda f_{1}$ is also coined the scaling factor.

The two interpretations of the FRT operation have been united into one formulation through a transformation kernel, as follows [8]:

$$
u_{p}(x)=\mathfrak{\Im}^{p}\left[u\left(x^{\prime}\right)\right](x)=\int_{-\infty}^{\infty} B_{p}\left(x, x^{\prime}\right) u\left(x^{\prime}\right) d x^{\prime},
$$

where $B_{p}\left(x, x^{\prime}\right)$ is the kernel of the transformation and $p$ is the fractional order. The kernel has two optical interpretations, one as propagation through GRIN medium [10]:

$$
\begin{gathered}
B_{p}\left(x, x^{\prime}\right) \\
=\sqrt{2} \exp \left[-\frac{x^{2}+x^{\prime 2}}{\omega}\right] \sum_{n=0}^{\infty} \frac{i^{-p n}}{2^{n} n !} H_{n}\left(\frac{\sqrt{2} x}{\omega}\right) H_{n}\left(\frac{\sqrt{2} x^{\prime}}{\omega}\right),
\end{gathered}
$$

and the other as a rotation operation applied over the Wigner plane [12]:

$$
B_{p}\left(x, x^{\prime}\right)=C_{1} \exp \left[i \pi \frac{x^{2}+x^{\prime 2}}{\lambda f_{1} \tan \phi}-2 \pi i \frac{x x^{\prime}}{\lambda f_{1} \sin \phi}\right],
$$

where $\omega$ is the coefficient that connects the two interpretations as given below:

$$
\omega=\sqrt{\lambda f_{1} / \pi}
$$


Careful examination of the expression of the FRT definition shows that the FRT is a localized transformation. In this context, "localized" means that the input function is actually multiplied by a space window as is done in the Gabor transform [13]. In the FRT case the space window is a phase window (the chirp phase function $\left.\exp \left[i \pi\left(x^{2}+x^{\prime 2}\right) /\left(\lambda f_{1} \tan \phi\right)\right]\right)$ rather than amplitude window as for the case of the original Gabor transform. In the case of the chirp function, increasing the distance from the origin results in an increase of the spatial frequency. Eventually, the spatial frequency becomes so high that while calculating the integral according to the FRT definition, under-sampling might occur. As a result higher frequencies are lost and the phase window is equivalent to an amplitude window.

\subsubsection{Properties of the FRT.}

(1) Linearity.

The FRT is a linear transformation. Thus,

$$
\begin{gathered}
\mathfrak{\Im}^{p}\left[c_{1} u_{1}(x)+c_{2} u_{2}(x)\right] \\
=c_{1} \mathfrak{\Im}^{p}\left[u_{1}(x)\right]+c_{2} \mathfrak{\Im}^{p}\left[u_{2}(x)\right],
\end{gathered}
$$

where $c_{1}, c_{2}$ are constants.

(2) Continuity.

Two FRTs with different orders $p_{1}$ and $p_{2}$ yield the following theorem:

$$
\begin{gathered}
\mathfrak{S}^{c_{1} p_{1}+c_{2} p_{2}}[u(x)]=\mathfrak{S}^{c_{1} p_{1}}\left[\mathfrak{S}^{c_{2} p_{2}}(u(x))\right] \\
=\mathfrak{S}^{c_{2} p_{2}}\left[\mathfrak{S}^{c_{1} p_{1}}(u(x))\right] .
\end{gathered}
$$

(3) Perseval's theorem.

$$
\int_{-\infty}^{\infty}\left|u_{0}\left(x_{0}\right)\right|^{2} d x_{0}=\int_{-\infty}^{\infty}\left|u_{p}\left(x_{p}\right)\right|^{2} d x_{p} .
$$

(4) Shift theorem.

If the input object is shifted by an amount of $a$, then its FRT yields

$$
\begin{gathered}
u_{p}(x ; a)=\mathfrak{\Im}^{p}\left[u_{0}\left(x_{0}+a\right)\right] \\
=\exp [i \pi a \sin \phi(2 x+a \cos \phi)] u_{p}(x+a \cos \phi) .
\end{gathered}
$$

(5) Scaling theorem.

If the input object is scaled by the factor of $a$, then its FRT yields

$$
\begin{gathered}
u_{p}(x ; a)=\mathfrak{\Im}^{p}\left[u_{0}\left(a x_{0}\right)\right] \\
=(\Phi / a) u_{\overline{\mathrm{p}}}\left(x / \sqrt{\sin ^{2} \phi\left(a^{2}+1 / a^{2}\right)}\right), \\
\bar{p}=(2 / \pi) \tan ^{-1}\left(a^{2} \tan \phi\right), \\
\Phi=\exp \left[i \pi x^{2}\left(a^{4}-1\right) /\left(a^{4} \tan \phi+1 / \tan \phi\right)\right] .
\end{gathered}
$$

\subsection{Fractional Correlation}

In several pattern recognition applications, the shift invariance property within the entire input plane is not necessary and may even be considered as a disadvantage. An example is the case where the object is to be recognized only when its location is inside a predefined window and rejected otherwise, e.g., a passport with a picture that should appear only at the upper right area. Several approaches for obtaining such space variance detection have been suggested. The first approach is the Tandem component processor that trades the shift invariance with high efficiency and high peak to correlation-energy ratio [14]. Different approach is based on coded phase processor that multiplexed many filters and yet kept the space bandwidth product $(S W)$ of the ordinary single filter correlator [15]. A space-variant Fresnel transform correlator [16], which is closely related to the lensless intensity correlator [17], was also suggested. A related approach is the tool coined fractional correlation (FC) whose optical implementation is done using a setup similar to the Vander Lugt correlator $[18,19]$. The FC operation allows controlling the amount of shift variant property of the correlation. This property is based on the shift variance of the FRT and it is more significant for the fractional orders of $p \approx 0+$ $2 N$ (imaging) and less for $p \approx 1+2 N$ (Fourier), where $N$ is an integer.

If we return again to the example of the passport picture, then opposed to a solution involving using an appropriate input pupil which is open in the desired location, the FC does not require any additional components for its optical implementation. Another example for the necessity of the FC is the case where the recognition should be based mainly on the central pixels and less on the edge pixels (for instance in systems whose spatial resolution is improved in the central pixels and, thus, the central region of pixels is more reliable for the recognition process). An important application for the FC might be the detection of localized objects using a single cell detector, eliminating the need for a CCD array detector. It may as well be used for efficient multiplexing of several filters with different processing tasks. The schematic sketch of the FC as well as an example of the optical setup that may be used for realizing it, are depicted in Fig. 2.

As can be seen, the process is based on obtaining the product of the fractional transforms of the distributions that are to be correlated and then rendering an additional FRT to obtain the final result. Analytically, the operation of $\mathrm{FC}$ of an input function, $f(x)$, with a reference pattern, $g(x)$, is defined as follows:

$$
C_{p_{1}, p_{2}, p_{3}}\left(x^{\prime}\right)=\mathfrak{\Im}^{p_{3}}\left\{\mathfrak{I}^{p_{1}}[f(x)] \times \mathfrak{\Im}^{p_{2}}[g(x)]\right\},
$$


(a)

(b)

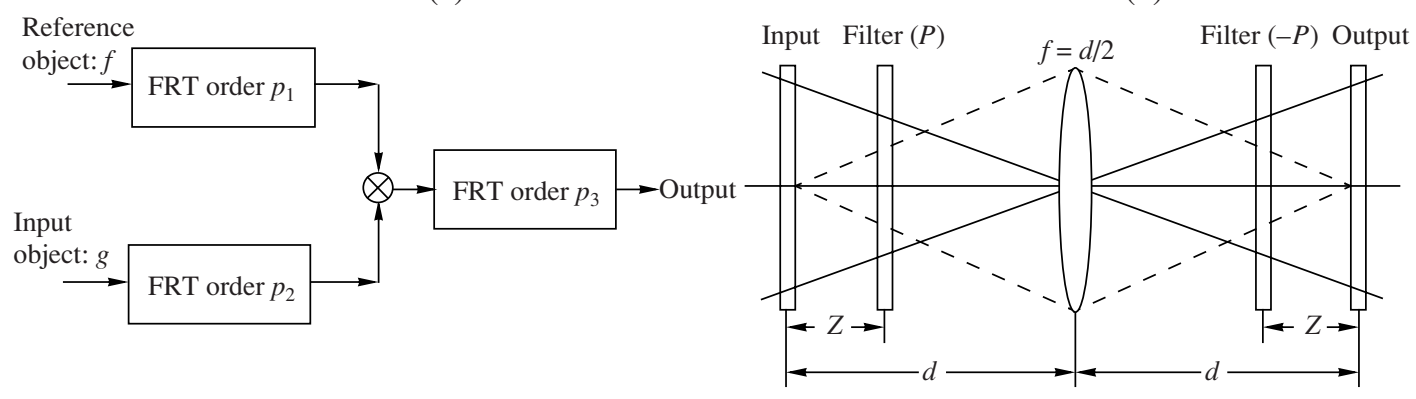

Fig. 2. (a) Schematic sketch for obtaining a generalized fractional correlation; (b) its experimental optical setup.

where $p_{1}, p_{2}, p_{3}$ are the orders of the FRTs. Due to various reasons, detailed in $[18,20]$, for optimization, the following relation should be realized:

$$
1 / \tan \phi_{1}+1 / \tan \phi_{2}+1 / \tan \phi_{3}=0,
$$

and the most obvious choice is

$$
p_{1}=p, \quad p_{2}=-p, \quad p_{3}=-1,
$$

where $p$ ranges from 0 to 1 . In this case, if the input coincides with the reference object, a perfect phase matching between the FRT of the object and the reference is obtained in the fractional domain. The inverse Fourier transform will just focus the resulting plane wave and produce a delta, i.e., a correlation peak.

An alternative optical implementation of the $\mathrm{FC}$ is based on illuminating, the object with a converging beam, as can be seen in Fig. 2b [21, 22]. This implementation permits the change of the convergence phase factor, multiplying the object, by displacing it along the optical axis. The matching between the distance-object filter and the convergence of the beam may produce any desired order and scaling factor. Hence, this approach is more convenient from the experimental point of view, since the exact dimensions of the input and filter transparencies are often not precisely determined. This is especially important for the case of using spatial light modulators (SLMs) for real time implementation of the filter. As the FRT is not exact there will be a quadratic phase factor multiplying the output plane. Therefore, the correlation plane will be displaced along the optical axis.

\section{GENERAL APPLICATIONS}

Besides the ability to change easily the space variance of the optical system, the FRT has shown to be very useful for many other signal processing applications. The main application is related to chirp noise removal. This application is based on the fact that if a chirp type noise of $\exp \left(-i a x^{2}\right)$ is fractionally Fourier transformed with the order of

$$
p=(2 / \pi) \tan ^{-1}(1 / a)
$$

the result is a delta function. Thus, in order to remove the noise, a simple notch filter may be placed in the proper FRT plane, using the fractional correlator configuration. Since the filter is a notch filter, the amount of loss signal's information is minimal. Another important application of the FRT is related to the fact that according to its second definition the FRT corresponds to the rotation of the Wigner chart by an angle of $p \pi / 2$. Thus, assuming that the Wigner chart for the signal and the noise distributions are as illustrated by Fig. 3a, one may see that filtering either in the Fourier plane (corresponds to projection of the Wigner chart over the $f_{x}$ axis) or filtering in the spatial plane (corresponds to projection of the Wigner chart over the $f_{x}$ axis), will result in partial loss of the signal's information. However, filtering in the proper FRT plane (the angle in the Wigner chart at which full separation exists between the projections of the signal and the noise) will allow full reconstruction of the signal by its noise [6]. Additional important application of the FRT is related to signal multiplexing. Due to the ability of the FRT to rotate the Wigner chart, the Wigner distribution of a signal may be arranged in a more efficient manner [8] as can be seen in Fig. 3b. This efficient arrangement saves additional bandwidth that may be needed for the transmission of the signal.

After performing this short overview of the basic fundamentals of the FRT, we will now describe the usage of this transformation for two new applications. The first one is related to blind source separation of images and the second one for RF photonics. Regarding blind source separation (BSS) there are many approaches, such as Principal Component Analysis (PCA), which uses second order statistics and decorrelates the outputs by using an orthogonal demixing matrix [23-25], algorithm that recovers images on the pixel-by-pixel basis [26] and approaches based upon adaptive filter that performs image separation in the 


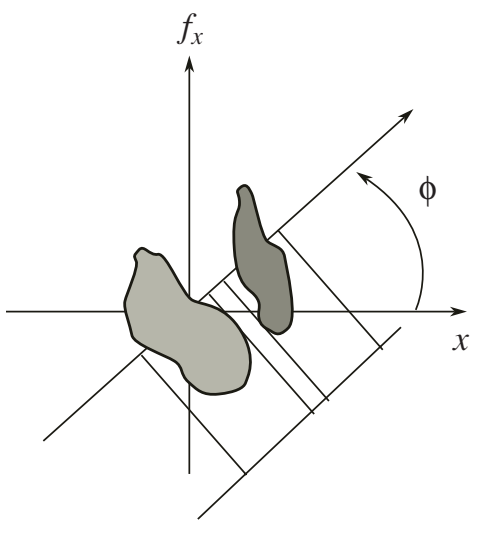

(a)

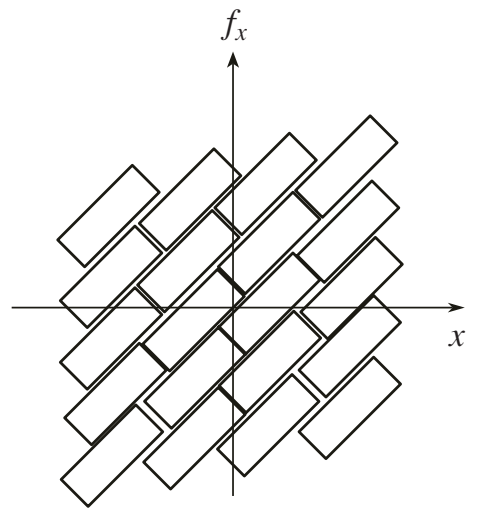

(b)

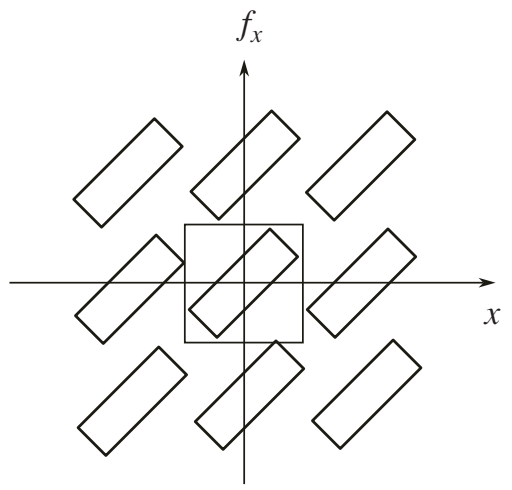

Fig. 3. (a) A Wigner chart of a signal and a noise where the FRT filtering is very applicable; (b) a Wigner chart of a signal that may be more efficiently multiplexed via an FRT.

Fourier space [27]. Another very common approach is called the "Block Gaussian likelihood" (BGL) algorithm suggested by D. T. Phalm and J. F. Cardoso [28]. We base our technique for images separation following an algorithm that seeks for the smallest fractional cross correlation [29] or the largest fractional auto correlation. RF photonics is a new evolving research field that deploys optical based processing techniques into RF and RADAR detection problems involving RF phased array sensors [30, 31] and filters [32]. Photonic RF filters have a lot of potential due to their capability to obtain high dynamic range, tunability and reconfiguration. Several configurations have been suggested employing highly dispersive fibers [33], fiber gratings [34], fiber optics prisms [35] or arrayed waveguide gratings (AWG) [36] or optical spectrum analyzers [37]. In this paper we present the usage of the FRT to separate between multiple chirped RF photonic signal.

\section{BLIND SOURCE SEPARATION OF IMAGES}

The novel algorithm that we have examined and which is based on the FRT managed to improve the blind source separation capability of mixed images [29].

The BSS process consists of recovering source images $s_{n}$ using only the observed data $\hat{M}$, where the source images and the mixing are unknown. The BSS process can be formulated by the linear mixing equation

$$
\hat{M}=A \hat{s},
$$

where $s_{n}, n=1 \ldots N$ are $N$ unknown independent uncorrelated noiseless image sources. These sources are instantaneously mixed with an unknown linear $N \times N$ matrix, $A$, which produces $N$ observation images $M_{n}$ $n=1 \ldots N$. The $N$ source images are to be uncorrelated noiseless images. The matrix $\hat{M}$ is a normalized matrix. Our basic assumptions to make this process work properly are

$$
\hat{M}_{n} \longrightarrow\left\langle\hat{M}_{n} \mid \hat{M}_{n}\right\rangle=1 \quad \text { and } \quad\left\langle M_{N}\right\rangle=0
$$

where $\langle\mid\rangle$ is a scalar product and \langle\rangle is the mean. In order to span the vectors space, which contains the mixtures $\hat{M}_{n}$ in a convenient manner, we define a new vector basis $V$. The vectors comprising this basis for the case of $N=2$ are obtained by the following linear combination:

$$
\begin{aligned}
& \hat{V}_{1}=\left(\hat{M}_{1}+\hat{M}_{2}\right) / \sqrt{2}, \\
& \hat{V}_{2}=\left(\hat{M}_{1}-\hat{M}_{2}\right) / \sqrt{2},
\end{aligned}
$$




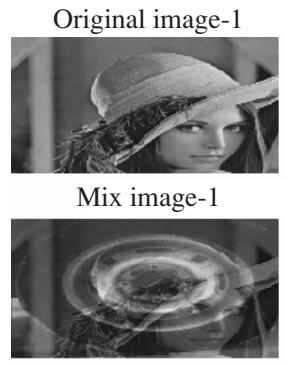

Separated image-S1a

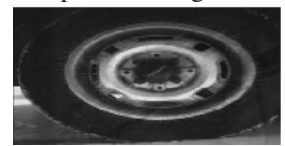

Original image-1

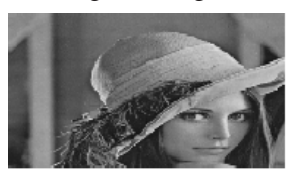

Mix image-1

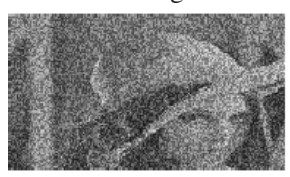

Separated image-S1a

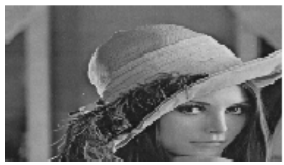

(a)
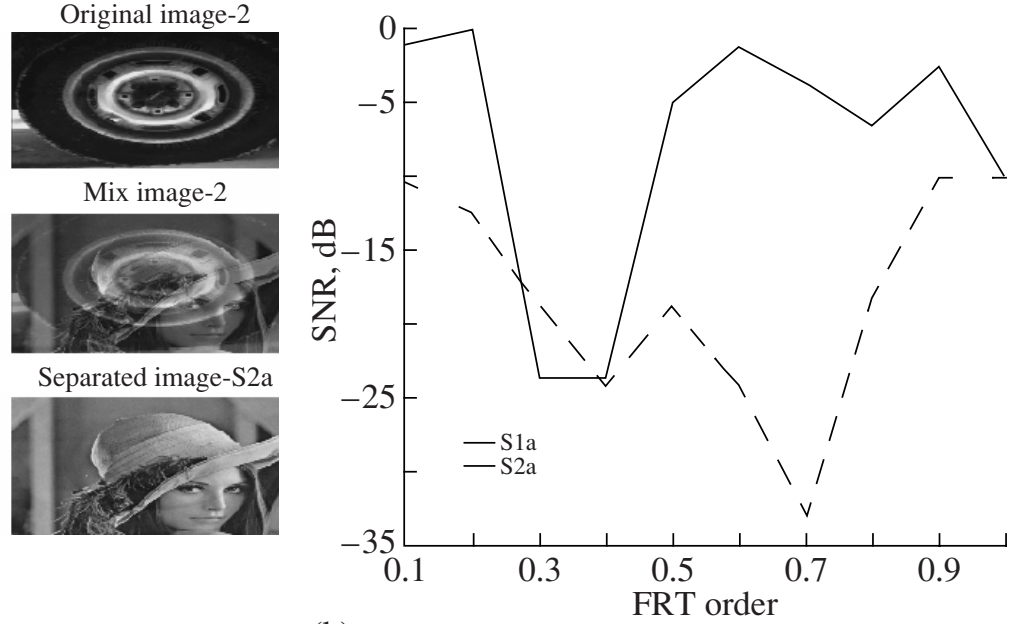

(b)
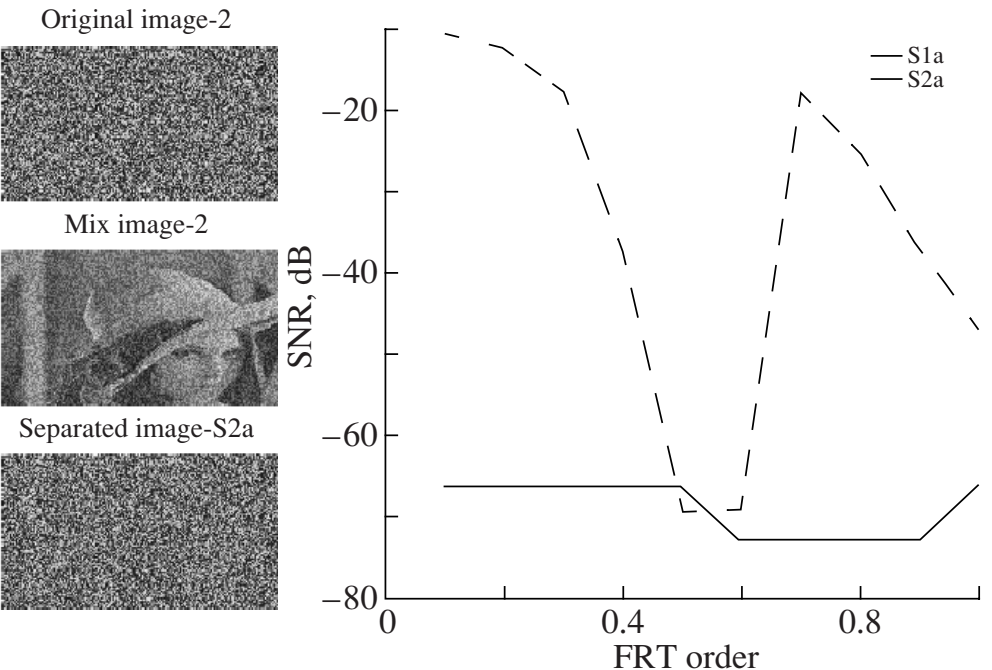

Fig. 4. Two numerical examples for blind source separation of images using fractional correlation. (a) Separation between LENA and a TIRE; $s_{1}$, dashed; $s_{2}$, solid; (b) Separation between LENA and noise; $s_{2}$, dashed; $s_{1}$, solid.

so that

$$
\left\langle\hat{V}_{p} \mid \hat{V}_{q}\right\rangle=\delta_{p q},
$$

where $\delta_{p q}$ is the Kronecker delta.

Each of the original images $s_{n}$ is a linear combination of the basis vector $V$, yet with an unknown coefficient's set. This argument may be stated in the following way:

$$
\begin{aligned}
& \hat{s}_{1}=\hat{V}_{1} \sin \phi_{1}+\hat{V}_{2} \cos \phi_{1}, \\
& \hat{s}_{2}=\hat{V}_{1} \sin \phi_{2}+\hat{V}_{2} \cos \phi_{2},
\end{aligned}
$$

where the parameters $\phi_{n}$ are to be determined.

The method by which the values of the parameters $\phi_{n}$ (corresponding to the original images) shall be found is as follows: any pair of images $s_{p}, s_{q}$ may be charac- terized by the FC function generally defined as in Eq. (23) [22]. We use fractional orders $p_{1}, p_{2}, p_{3}$, with the values of: $p_{1}=p, p_{2}=-p, p_{3}=-1$, where $p$ is ranging from 0 to 1 . We search for the minimal cross correlation function for all values of the parameter $\phi_{n}$ and for all values of the fractional order $p$. Allocating such values provides us with the parameter $\phi_{n}$ and, therefore, with the separated images $s_{n}$.

Here are some numerical simulations. As seen in Fig. 4a, two mixed images of LENA and TIRE were separated. The left part of Fig. 4a presents the separation results of the two images for FRT order of 0.7 and the right part displays the SNR as function of the fractional order. Figure $4 \mathrm{~b}$ presents additional simulations in which the LENA image was separated from random noise. Once again the left part of the figure presents the separation results of the two mages for FRT order of 0.6 
(a)

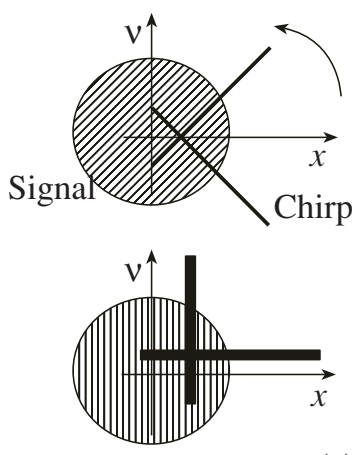

(c)

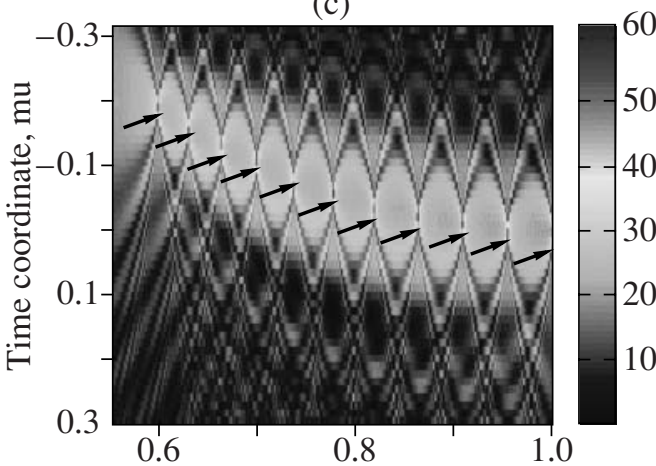

(e)

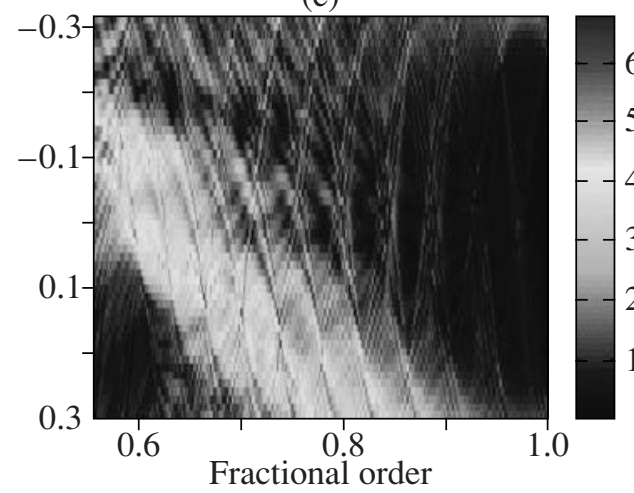

(b)

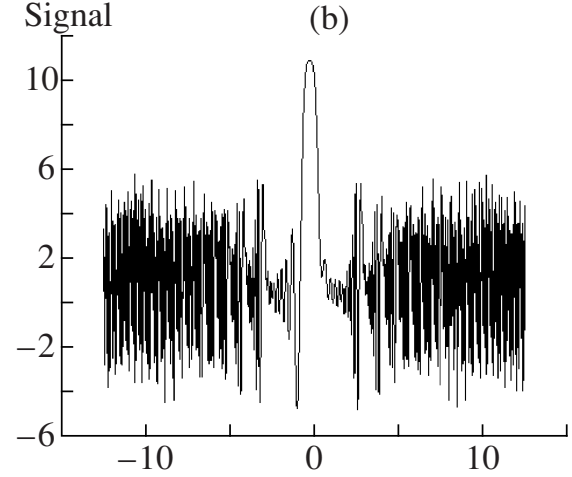

(d)

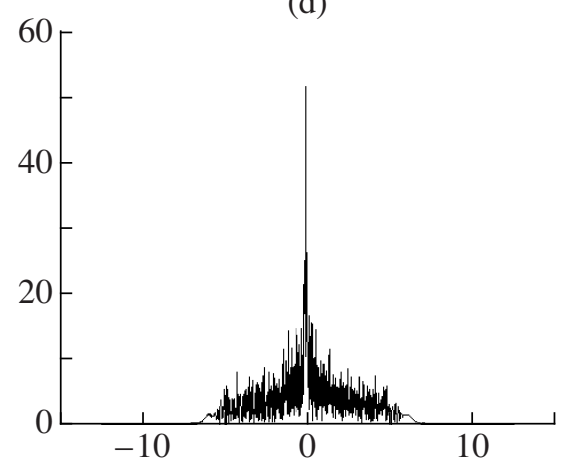

(f)

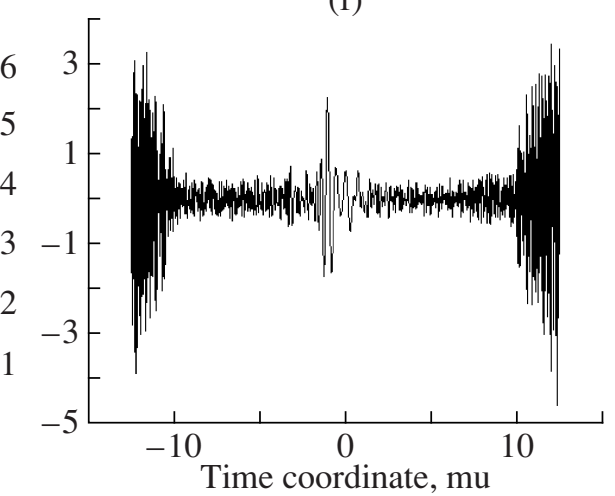

Fig. 5. (a) Detection of several chirps: schematic sketch; (b) the input signal consisting out of 11 chirps; (c) the $x-p$ chart; (d) the peak obtained at one of the fractional order where one of the chirps becomes a delta function; (e) the $x-p$ chart after filtering the 11 chirps; (f) the signal in the time domain.

and the right part displays the SNR as function of the fractional order. Just for comparison the common $B G L$ algorithm obtained SNR of only approximately $-10 \mathrm{~dB}$ for the mixture between the LENA and the TIRE images.

\section{RF-PHOTONICS RELATED APPLICATIONS}

RF photonics is a field in which RF signals are processed with optical modules to obtain simplicity and performance. In synthetic aperture radars (SAR), one sends chirp signals towards objects and then receives it at the receiver. In case where there are several chirps sent towards several potential targets, the secondary reflections from the first object may mix with the first reflection from the second object etc. In addition sometimes there is a desire to map wide field of view and, thus, to multiplex and to transmit several RF chirp signals simultaneously. In both cases a great advantage can be obtained if the antenna will transmit several chirps each having different temporal starting position and spectral specification and yet at the receiver those different chirps could be separated efficiently without damaging the information of the other chirps. To do that, we suggest using the $x-p$ chart [22] and to apply filtering in the FRT domain. The $x-p$ chart of a signal is a chart in which one axis is the various fractional orders and the other axis is the FRT distribution that is obtained for those orders. For explaining, let us start with an example where two different chirps are added 
to a general signal. Its Wigner space could be as depicted in Fig. 5a where the two different chirps are the two lines. By applying FRT with proper order we may rotate the Wigner space such that the first chirp could be filtered out (as depicted in the lower part of Fig. 5a). The chirps can be filtered out when they become horizontal line since then their projection over the frequency axis is compressed into a single frequency (a delta function). Then, by applying additional FRT with different fractional order, the Wigner space could be rotated again such that the second chirp will become a horizontal line. Thus, by cascading several FRT filtering systems that will process the RF signals one may deal with detection of several chirps while their number does not exceed the number of the cascaded systems. Note that the RF signals can be processed by optical modules if the detected RF signal is modulated on an optical carrier.

Figures $5 \mathrm{~b}-5 \mathrm{f}$ present several numerical simulations. In Fig. 5b, 11 different chirps were summed together in time window of $25 \mu \mathrm{s}$ and bandwidth of $15 \mathrm{MHz}$. Each chirp had a different temporal starting points and different bandwidth. In Fig. 5b, one may see the temporal distribution of the summed signals. Figure $5 \mathrm{c}$ presents its $x-p$ chart. In that chart, one may see several points of focus (delta functions) while each point corresponds to the parameters of a different chirp signal. The focusing points are marked by arrows in Fig. 5c. The mathematical relation between the order at which the delta function is obtained and its temporal position, in the case where the chirp function expressed as $\exp \left[-i \pi \alpha\left(t-t_{0}\right)^{2}\right]$, is as follows:

$$
p_{0}=\left[2 \tan ^{-1}(1 / \alpha)\right] / \pi, \quad t^{\prime}=t_{0} \sin (\pi p / 2),
$$

where $p_{0}$ is the fractional order at which the delta function is obtained and $t^{\prime}$ is its temporal position while $t_{0}$ was the position of the center of the original chirp. Figure $5 \mathrm{~d}$ presents how each plane of the focusing point looks like prior to the notch filtering procedure. The delta function obtained at that specific plane corresponds to a single chirp function whose parameters match the applied fractional order as described by Eq. (32). The rest of the chirps are spread all over the temporal window and thus their energy is not damaged when a notch filters out the delta function of that single predetermined chirp. The result obtained after the notch filtering is seen in Figs. 5e-5f. The filtering procedure consists out of performing FRT with fractional order that corresponds to the focusing points, then filtering the delta function using notch filter and then performing an inverse FRT. This operation is repeated eleven times for all the chirps in the summed signal. In Fig. 5e, one may see the $x-p$ chart after filtering all the eleven chirps and, in Fig. 5f, we present the temporal behavior of the remaining components. As seen from Fig. 5e, the chirps were filtered out and no focusing points were remained.

\section{ACKNOWLEDGMENTS}

The authors would like to acknowledge the contribution of Noam Shamir and Shlomo Zach to the formulation of this manuscript.

\section{REFERENCES}

1. G. S. Agarwal and R. Simon, Opt. Commun. 110, 23 (1994).

2. L. B. Almeida, An Introduction to the Angular Fourier Transform (IEEE-IC ASSP, Minneapolis, 1993).

3. L. B. Almeida, IEEE Trans. Signal Proc. 42, 3084 (1994).

4. L. M. Bernardo and O. D. D. Soares, Opt. Commun. 110, 517 (1994).

5. E. U. Condon, Proc. Nat. Acad. Sci. USA 23, 158 (1937).

6. R. G. Dorsch, A. W. Lohmann, Y. Bitran, et al., Appl. Opt. 33, 7599 (1994).

7. D. Mendlovic, M. Ozaktas, and A. W. Lohmann, Appl. Opt. 33, 6188 (1994).

8. H. M. Ozaktas, B. Barshan, D. Mendlovic, and L. Onural, J. Opt. Soc. Am. A 11, 547 (1994).

9. D. Mendlovic and H. M. Ozaktas, J. Opt. Soc. Am. A 10, 1875 (1993).

10. H. M. Ozaktas and D. Mendlovic. J. Opt. Soc. Am. A 10, 2522 (1993).

11. H. M. Ozaktas and D. Mendlovic, Opt. Commun. 101, 163 (1993).

12. A. W. Lohmann, J. Opt. Soc. Am. A 10, 2181 (1993).

13. D. Gabor, J. Inst. Elec. Eng. 93, 429 (1946).

14. H. O. Bartelt, Appl. Opt. 24, 3811 (1985).

15. J. R. Leger and S. H. Lee, Appl. Opt. 21, 274 (1982).

16. J. A. Davis, D. M. Cottrell, N. Nestorovic, and S. M. Highnote, Appl. Opt. 31, 6889 (1992).

17. G. G. Mu, X. M. Wang, and Z. Q. Wang, Proc. Soc. Photo-Opt. Instrum. Eng. 673, 546 (1986).

18. D. Mendlovic, H. M. Ozaktas, and A. W. Lohmann, Appl. Opt. 34, 303 (1995).

19. D. Mendlovic, Y. Bitran, R. G. Dorsch, and A. W. Lohmann, J. Opt. Soc. Am. A 12, 1655 (1995).

20. A. W. Lohmann, Z. Zalevsky, and D. Mendlovic, Opt. Commun. 128, 199 (1996).

21. J. Garcia, R. Dorsch, A. W. Lohmann, C. Ferreira, and Z. Zalevsky, Opt. Commun. 133, 393 (1997).

22. H. M. Ozaktas, Z. Zalevsky, and M. A. Kutay, The Fractional Fourier Transform with Applications in Optics and Signal Processing (Wiley, New York, 2001).

23. A. Hyvärinen and E. Oja, Neural Networks 13, 411 (2000).

24. T. P. Jung, S. Making, M. J. Mckeown, et al., Proc. IEEE, 89, 1107 (2001).

25. E. Oja and M. Plumbley, in Proceedings of the 4th International Symposium on Independent Component Analysis and Blind Signal Separation (Nara, Japan, 2003).

26. H. Szu and I. Koperiva, in Proceedings of the 4th International Symposium on Independent Component Analysis and Blind Signal Separation (Nara, Japan, 2003). 
27. W. Kaprzak and A. Okazaki, in Proceedings of the 4th International Symposium on Independent Component Analysis and Blind Signal Separation (Nara, Japan, 2003).

28. A. Belouchrani and M. G. Amin, IEEE Trans. Signal Proc. 46, 2888 (1998).

29. N. Shamir, Z. Zalevsky, and D. Mendlovic, in Proceedings of the 7th International Conference on Optoelectronics, Fiber Optics, and Photonics (Cochin, India, 2004).

30. H. R. Fetterman, Y. Chang, D. C. Scott, et al., IEEE Microwave and Guided Wave Lett. 5, 414 (1995).

31. L. Xu, R. Taylor, and S. R. Forrest, IEEE Photon. Technol. Lett. 8, 160 (1996).
32. R. A. Minasian and D. B. Hunter, in Proceedings of the Conference on Optical Fiber Communication (OFC'97) (Dallas, USA, 1997), ThH3, pp. 339-340.

33. D. Norton, S. Johns, C. Keefer, and R. Soref, IEEE Photon. Technol. Lett. 6, 831 (1994).

34. J. Campany, D. Pastor, and B. Ortega, IEEE Trans. Microwave Theory Tech. 47, 1321 (1999).

35. M. Y. Frankel and R. D. Esman, IEEE Photon. Technol. Lett. 7, 191 (1995).

36. F. Coppinger, S. Yegnanarayanan, P. D. Trinh, and B. Jalali, IEEE Photon. Technol. Lett. 9, 339 (1997).

37. Z. Zalevsky, A. Shemer, V. Eckhouse, D. Mendlovic, and S. Zach, J. Opt. Soc. Am. A 22, 1668 (2005). 